\title{
Breakdown of the Dissipationless Quantum Hall State: Quantised Steps and Analogies with Classical and Quantum Fluid Dynamics
}

\author{
L. EAVES
}

School of Physics and Astronomy, University of Nottingham

Nottingham NG7 2RD, UK

\begin{abstract}
The breakdown of the integer quantum Hall effect at high currents sometimes occurs as a series of regular steps in the dissipative voltage drop measured along the Hall bar. The steps were first seen clearly in two Hall bars used to maintain the US Resistance Standard, but have also been reported in other devices. It is proposed that the origin of the steps can be understood in terms of an instability in the dissipationless flow at high electron drift velocities. The instability is induced by impurity- or defect-related inter-Landau level scattering processes in local microscopic regions of the Hall bar. Electron-hole pairs (magneto-excitons) are generated in the quantum Hall fluid in these regions and that the electronic motion can be envisaged as a quantum analogue of the von Karman vortex street which forms when a classical fluid flows past an obstacle.
\end{abstract}

PACS numbers: 73.43.Jn, 73.43.Cd, 73.61.Ey

\section{Introduction}

In the integer quantum Hall effect (QHE) [1], a two-dimensional electron fluid carries an almost dissipationless current and the ratio of the current to the Hall voltage is quantised in units of $e^{2} / h$. This quantisation is so precise that it is used to define the unit of electrical resistance. Above a critical value of current, the dissipative voltage, $V_{x}$, measured along the direction of current flow, increases rapidly, leading to quantum Hall effect breakdown. The microscopic mechanism, or mechanisms, responsible for breakdown have been a topic of interest and controversy for almost twenty years [2]. 
In two samples used to maintain the US Resistance Standard [3, 4], Cage and co-workers observed a staircase of ten or more steps in the longitudinal voltage, $V_{x}$, at breakdown as shown in Fig. 1 . The steps have a regular height, $\Delta V_{x} \approx 5 \mathrm{mV}$ and are accompanied by hysteresis and intermittent noise. The data were obtained by measuring $V_{x}$ with a large current $I$ flowing along the length of a bar-shaped sample (see Fig. 1a, inset) made from a GaAs/(AlGa)As semiconductor heterostructure. The figure shows sweeps of the applied magnetic field $B$ over an extended range around $12 \mathrm{~T}$ at which the Landau level filling factor $\nu=2$. Despite the extensive literature on QHE breakdown, reviewed in Ref. [2], the precise origin of the steps has remained uncertain. In particular, they are not readily explicable in terms of the properties of the magneto-conductivity tensor components, $\sigma_{x x}$ and $\sigma_{x y}$, which describe successfully most of the macroscopic properties of the quantum Hall fluid (QHF).
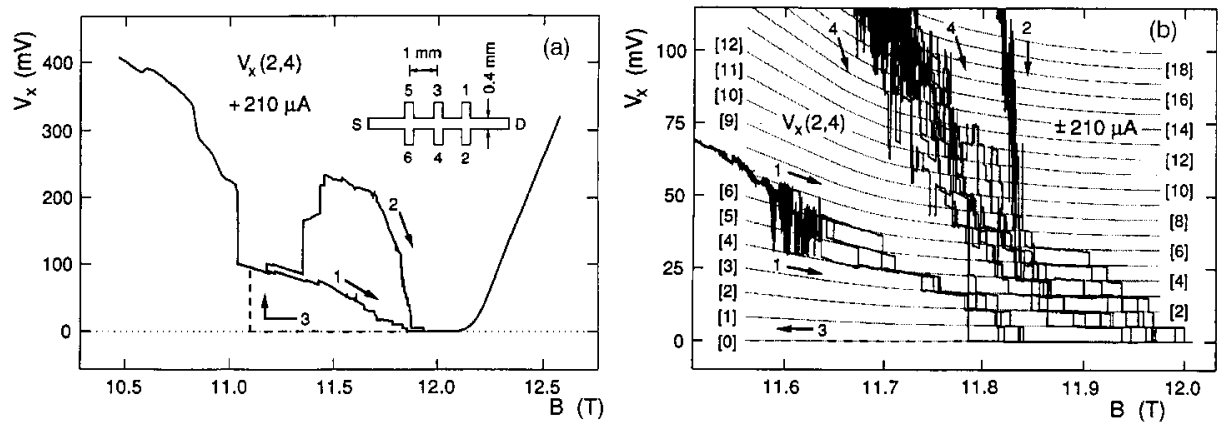

Fig. 1. QHE breakdown in one of the Hall bar samples used to maintain the US Resistance Standard at the National Institute of Standards and Technology, Gaithersburg. (a) Plots of longitudinal voltage $V_{x}$ versus magnetic field $B$ at $T=1.3 \mathrm{~K}$, in the region of the $\nu=2$ filling factor, $I=210 \mu \mathrm{A}$. The arrows show the hysteresis for various sweeps of $B$. (b) A detail of the breakdown curve, showing the large number of steps in $V_{x}$ (both figures from Ref. [3], courtesy of NIST).

QHE breakdown corresponds to the disruption of dissipationless electron flow along the voltage equipotentials and to an abrupt increase in $V_{x}$ from a very low value. The dissipative current, $i(\ll I)$, flowing perpendicular to the equipotentials is given by $2 e^{2} V_{x} / h$. Such a current can arise from inter-Landau level tunnelling [5-10]. In this process, an electron in the lower filled Landau level scatters elastically (or quasi-elastically with the emission of a low energy acoustic phonon) into the empty state of the upper unfilled Landau level. Since the wave functions of the Landau states are strongly localised along the direction perpendicular to the equipotentials and have strongly decaying Gaussian tails, a large electric field is required for significant spatial overlap between the initial and final states of the same energy, i.e. the electric field $E$ must be $\sim \hbar \omega_{\mathrm{c}} / e \ell_{\mathrm{B}}$, where $\omega_{\mathrm{c}}=\mathrm{eB} / \mathrm{m}$ 
is the cyclotron frequency, $m$ is the electron effective mass $\left(=0.07 m_{\mathrm{e}}\right.$ for $\left.\mathrm{GaAs}\right)$ and $\ell_{\mathrm{B}}=(\hbar / e B)^{1 / 2}$ is the magnetic length. Alternatively, this condition can be expressed in the form $m v_{\mathrm{d}}^{2} \sim \hbar \omega_{\mathrm{c}}$, where $v_{\mathrm{d}}=E / B$ is the drift speed of the cyclotron orbit centre. For the experimental conditions of Fig. 1, the average value of the component of the electric field $E$ along $y$ (the average Hall field) is relatively small, $\bar{E}_{y}=V_{\mathrm{H}} / w \approx 7 \times 10^{3} \mathrm{~V} \mathrm{~m}^{-1}$, almost two orders of magnitude too small to produce significant wave function overlap.

The essence of the model proposed here is that $E$ and hence $v_{\mathrm{d}}$ can be much larger in localised microscopic regions of the Hall bar. These large values of $E$ can arise due to the presence of charged impurities and/or the proximity of the edges of the Hall bar. In these regions the QHF is unstable against inter-Landau level tunnelling. This effect can be envisaged as a quantum analogue of the generation of vortex-pairs in a von Karman vortex street, when a classical fluid flows past an obstacle at sufficiently high speed at Reynold's numbers $\operatorname{Re} \geq 10^{2}$, and may also be analogous to vortex pair formation in superfluids.

\section{Model and comparison with experiment}

For simplicity, let us assume a quadratic dependence of the electron potential energy $\phi$ in the local region, as shown schematically in Fig. 2a. Outside this region, the Hall field is smaller. Over the distance $s$ along the $y$-direction, we write $e \phi \approx m \omega_{0}^{2} y^{2} / 2$. This model potential is a function of only one unknown parameter $\omega_{0}$, and corresponds to a large local velocity gradient $\Omega=\mathrm{d} v_{x} / \mathrm{dy}=$ $\left(\mathrm{d}^{2} \phi / \mathrm{d} y^{2}\right) / B=\omega_{0}^{2} / \omega_{\mathrm{c}}$. The equipotentials shown in Fig. $2 \mathrm{~b}$ give rise to this type of velocity gradient. Due to the character of electron motion in crossed electric and magnetic fields, they also correspond to the directions of dissipationless flow in this region. A potential distribution of this kind, where $E$ is locally much larger than $\bar{E}_{y}$, can arise from the combined effect of a charged donor impurity located current close to the QHF, and the large Hall field due to the charge distribution near the edge of the sample $[9,11-13]$. These charges are essentially unscreened by the presence of the dissipationless QHF [13, 14].

Figure $2 \mathrm{a}$ also shows the wave function of a state $\alpha_{0}$ in the lowest filled Landau level $(n=0)$ and of a state $\beta_{1}$ in the $n=1$ level. The two states have the same energy (the dispersion of the Landau level energies is due, of course, to the spatial variation of $\phi$ ). For simplicity, we neglect spin. The orbit centres of $\alpha_{0}$ and $\beta_{1}$ are separated by a distance $\Delta y=s$, given by

$$
m \omega_{0}^{2} s^{2} / 2 \approx \hbar \omega_{c}
$$

For small $\omega_{0}$, spatial overlap between the wave functions is negligible. However, if $\omega_{0}$ is sufficiently large, overlap occurs (see figure) and an electron in state $\alpha_{0}$ can undergo a quantum transition to the unfilled state $\beta_{1}$. This effectively creates a magneto-exciton [15] in the QHF. The electron (upper Landau level) and 


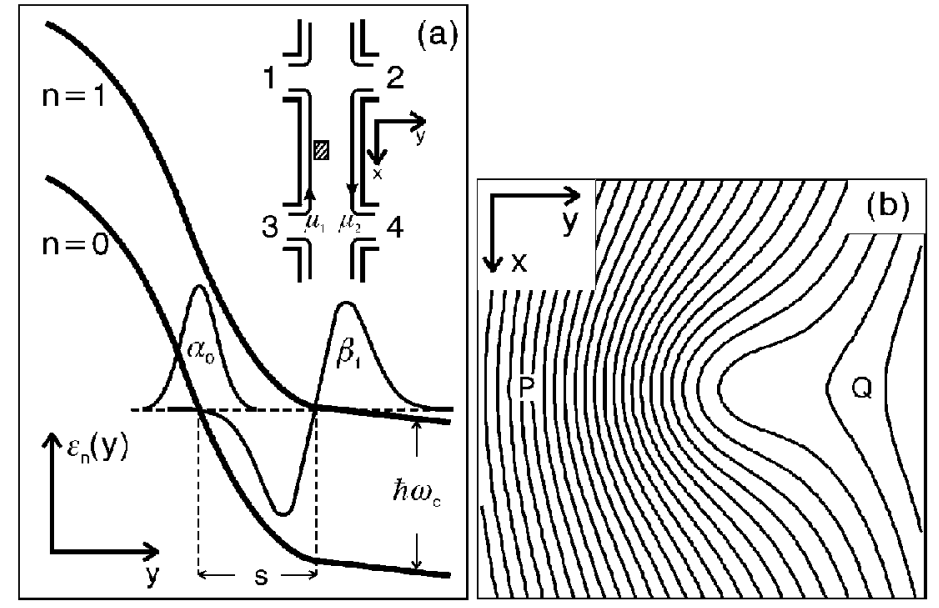

Fig. 2. (a) Model for spatial variation of energy of the two lowest Landau levels, $n=0$ and $n=1$ in a localised region of QHE breakdown. The eigenfunctions $\alpha_{0}$ and $\beta_{1}$ are in the $n=0$ (filled) and $n=1$ (empty) Landau levels respectively, at filling factor $\nu=2$. They have the same energy and spatially overlap each other. The length $s$ is the distance over which the cyclotron orbit centre jumps in an inter-Landau level tunnelling transition. The inset shows schematically the geometry of the Hall bar sample. $V_{x}$ is measured between probes 1 and 3 . (The Hall voltage $V_{\mathrm{H}}$ is measured across 1 and 2 or 3 and 4.) The small cross-hatched region indicates schematically a breakdown region near the edge of the sample. The difference between chemical potentials at the two edges, $\mu_{1}-\mu_{2}=e V_{\mathrm{H}}$. (b) Plot of the voltage equipotentials in the plane of the quantum fluid for a region of large velocity gradient in which breakdown occurs. The energy variation of the Landau levels shown in box (a) above corresponds to that along the line PQ.

hole (lower Landau level) which form the magneto-exciton can break apart and scatter in opposite directions along the direction of the Hall electric field. Thus an inter-Landau level transition of this kind leads to the dissipative breakdown current, $i$. The scattering process requires a jump of orbit centre position which can be induced by the presence of the charged impurity which destroys translational symmetry.

To compare our model with the data, in particular with the measured value of $\Delta V_{x}$, we must determine the size of dissipative current $i$ generated by inter-Landau level tunnelling. In principle, the tunnelling rate could be calculated using Fermi's golden rule. The problem is that inter-Landau level tunnelling creates an electron in the upper, previously empty, Landau level and an empty state, or hole, in the previously filled lower Landau level. The two particles experience a Coulombic attractive interaction and also tend to screen the local potential which generated them, thereby perturbing the drift motion of other electrons in adjacent states 
of the lowest Landau level. Since the interaction between the electron-hole pair (or magneto-exciton) and the QHF is quite complicated, even in the absence of impurity or edge charges [15], we cannot make a calculation based on a simple independent particle picture. To avoid this difficulty, let us consider the following semiclassical description, which seems to account for the essential features of the breakdown mechanism. The description draws on an analogy with vortex shedding in classical hydrodynamics and effectively uses a dimensional argument to estimate the rate of electron-hole pair formation at a given breakdown site.

At the critical breakdown values of current and magnetic field, we assume that the electric field in the local breakdown region is just large enough to induce inter-Landau level tunnelling. This process can be envisaged as electron-hole pair formation, the electron occupying state $\beta_{1}$ and the hole corresponding to the electron missing from state $\alpha_{0}$ in the otherwise filled lower Landau level. The presence of the electron-hole pair acts to screen the locally strong electric field, thus temporarily inhibiting further generation of pairs by tunnelling. However, due to the presence of the local electric field, the electron-hole pair drift away from the region in which they are formed. For the potential distribution shown in Fig. 1, assuming an independent particle description, the hole left in state $\alpha_{0}$ has a high $\boldsymbol{E} \times \boldsymbol{B}$ drift velocity, whereas the electron in $\beta_{1}$ has a lower drift velocity, each given by the local value of the electric field. We account qualitatively for the effect of the Coulombic attraction between the electron and hole by assuming that the pair drift at a speed determined by the mean electric field, which is $m \omega_{0}^{2} s / 2 e$. The mean drift speed is therefore $\left\langle v_{\mathrm{d}}\right\rangle \approx \Omega s / 2$ and the pair move a distance $\approx s$ from the formation region in a time $\tau=2 / \Omega \gamma$, where $\gamma$ is a numerical factor, $\approx 1$. At this separation, they no longer screen effectively the strong local electric field, so this is restored, leading to the creation of another electron-hole pair. Assuming that the time $\tau$ is the controlling factor in the formation of electron-hole pairs, we can take the rate of generation to be $\approx \gamma \Omega / 2$. On a longer timescale, given by the intra-Landau level energy relaxation time $\tau_{\varepsilon} \approx 10^{-10} \mathrm{~s}$, we assume that the electron-hole pair breaks up, the two particles diffusing away from each other with velocity components perpendicular to the equipotentials [16]. This process can occur for each particle by emission of low energy acoustic phonons through intra-Landau level scattering processes. The dissipative backscattering current $i \approx e \gamma \Omega / 2$ causes an incremental increase in $V_{x}$ given by

$$
\Delta V_{x}=h i / 2 e^{2}=h \gamma \Omega / 4 e .
$$

The steps shown in Fig. 1 would then correspond to successive contributions to the dissipative current of other breakdown regions with a similar local potential distribution, each adding $\approx e \gamma \Omega / 2$ to the dissipative current as $B$ is swept away from the $\nu=2$ value. If we assume that the background impurity density in the NIST sample is $\approx 10^{20} \mathrm{~m}^{-3}$, we can estimate the number, $N_{\mathrm{c}}$, of charged impurities which occupy the high electric field region close to the edge of the Hall bar. The two voltage probes which measure $V_{x}$ are separated by $1 \mathrm{~mm}$. Let us also assume 
that the high electric field region close to the edge is $\approx 10^{-7} \mathrm{~m}$ wide and that the charged scattering centres are within $5 \mathrm{~nm}$ of the central plane of the QHF. We then obtain $N_{\mathrm{c}} \approx 50$, which is roughly consistent with the total number of steps observed in the NIST experiment.

To test the model, we now compare the observed value of $\Delta V_{x}$ with the value of $\omega_{0}$ required to generate inter-Landau level tunnelling. Using Eq. (1) and recalling that $\Omega=\omega_{0}^{2} / \omega_{\mathrm{c}}$, we write Eq. (2) in the form

$$
e \Delta V_{x}=\pi \gamma \hbar^{2} / m s^{2} \text {. }
$$

This equation relates the observed value of $\Delta V_{x}=5 \mathrm{mV}$ to the orbit centre separation, $s$, rather than to $\omega_{0}$. Setting $\gamma \approx 1$, we then obtain $s^{2} / \ell_{\mathrm{B}}{ }^{2} \approx \pi \hbar \omega_{\mathrm{c}} / e \Delta V_{x}$, which gives $s \approx 3.5 \ell_{\mathrm{B}}$, where $\ell_{\mathrm{B}}=(\hbar / e B)^{1 / 2}$. This value of $s$ agrees quite well with the condition for alignment of the classical turning points of the wave functions $\alpha_{0}$ and $\beta_{1}$, namely when $s_{\mathrm{TP}}=(\sqrt{3}+1) \ell_{\mathrm{B}}$. This condition corresponds to the threshold of the strong increase in the matrix element for inter-Landau level transitions [10].

The model can also be tested against the recently-reported voltage steps for $\mathrm{QH}$ breakdown of hole gases [17]. Here the typical step height is $\Delta V_{x} \approx 1 \mathrm{mV}$ for breakdown at $\nu=2$. Using Eq. (3) and the measured value of the hole effective mass, $m_{\mathrm{h}}=0.15 m_{\mathrm{e}}[18]$, we obtain $s=2.3 \ell_{\mathrm{B}}$, which is also quite close to the value of $s_{\mathrm{TP}}$. The hole gas data therefore provide further support for the model.

Recalling that $\Omega=\omega_{0}^{2} / \omega_{\mathrm{c}}$ we can use Eq. (2) to confirm that the size of $\omega_{0}$ required for breakdown is consistent with that produced by an unscreened charged impurity. For such an impurity located at a distance $\Delta z$ from an idealised strictly 2-dimensional QHF, it is easily shown that a step height of $\Delta V_{x}=$ $5 \mathrm{mV}$ observed at $B \approx 12 \mathrm{~T}$ by Cage et al. corresponds to a value of $\Delta z$ given by $\left(h / 16 \pi \varepsilon_{\mathrm{r}} \varepsilon_{0} B \Delta V_{x}\right)^{1 / 3} \approx 13 \mathrm{~nm}$, where $\varepsilon_{\mathrm{r}} \approx 12$ is the permittivity of GaAs. This value corresponds very closely to the mean distance of the QHF from the GaAs/(AlGa)As interface [19] and indicates that charged impurities close to the interface can generate the local velocity required for our breakdown model.

\section{Analogies with classical and quantum fluids}

The proposed mechanism for the dissipative current $i$ is closely analogous to the formation of vortex-antivortex pairs which are created when a classical fluid flows past an obstacle, e.g. a cylinder, above a critical Reynolds number, $\operatorname{Re} \approx 60$. The formation of an electron-hole pair in an almost dissipationless QHF due to inter-Landau level tunnelling in the vicinity of a charged impurity then corresponds to the gain of an electron (vortex) by the previously empty $n=1$ Landau level and the loss of an electron (or, creation of hole = gain of an antivortex) by the previously filled lower Landau level. In this picture, the creation of vortices of opposite sign, but equal magnitude, in the upper and lower Landau levels can be compared with the conservation of circulation (vorticity) in inviscid classical fluid. 
There are also obvious analogies with the creation of vortices in quantum fluids such as atomic Bose condensates and superfluids.

Recently, the author (L.E.), in collaboration with Dr. A.M. Martin and Dr. K.A. Benedict, has developed further the model proposed here. We have calculated the magneto-exciton (also known as magneto-plasmon) modes of a QHF in the presence of a strong electric field. The model is based on early work of Kallin and Halperin [15] and of MacDonald et al. [20]. We have identified the critical electric field for breakdown, which is close to the value obtained using the simple arguments described in this paper. We are also able to calculate the rate of magneto-exciton formation at a given impurity site which gives a value of the dissipative voltage step height, which is quite close to that given by Eq. (3). This work is currently submitted for publication [21].

\section{Summary and future work}

A model has been proposed to explain the voltage steps observed in the breakdown of the QHE in two samples used to maintain the US Resistance Standard. The model suggests that we can view this type of breakdown as an instability in the flow of the QHF. This instability arises from the generation of electron-hole pairs by inter-Landau level tunnelling in local regions of the Hall bar, where the electric field is large. This type of process has analogies with vortex pair formation in classical fluids and superfluids.

\section{Acknowledgments}

I am grateful to Dr. M. Cage and NIST for permission to use Fig. 1, and to my colleagues F.W. Sheard, K.A. Benedict and A.M. Martin for helpful discussions. This work was partly supported by the Engineering and Physical Sciences Research Council (UK).

\section{References}

[1] K. von Klitzing, G. Dorda, M. Pepper, Phys. Rev. Lett. 45, 494 (1980).

[2] For a comprehensive review of QHE breakdown, see G. Nachtwei, Physica E 4, 79 (1991).

[3] M.E. Cage, J. Res. Natl. Inst. Stand. Technol. 98, 361 (1993). This reference, and Ref. [4] below, describe the voltage steps observed in the two US Resistance Standard samples with reference numbers $\operatorname{GaAs}(7)$ and $\operatorname{GaAs}(8)$.

[4] C.F. Lavine, M.E. Cage, R.E. Elmquist, J. Res. Natl. Inst. Stand. Technol. 99, 757 (1994).

[5] H.L. Störmer, A.M. Chang, D.C. Tsui, J.C.M. Hwang, in: Proc. 17th Int. Conf. on the Physics of Semiconductors, 17th ICPS, San Francisco 1984, Eds. J.D. Chadhi, W.A. Harrison, Springer, Berlin 1985, p. 267. 
[6] O. Heinonen, P.L. Taylor, S.M. Girvin, Phys. Rev. 30, 3016 (1984).

[7] L. Eaves, P.S.S. Guimarães, J.-C. Portal, J. Phys. C, Solid State Phys. 17, 6177 (1984).

[8] L. Eaves, F.W. Sheard, Semicond. Sci. Technol. 1, 346 (1986).

[9] N.Q. Balaban, U. Meirav, H. Shtrikman, Y. Levinson, Phys. Rev. Lett. 71, 1443 (1993).

[10] C. Chaubet, A. Raymond, D. Dur, Phys. Rev. B 52, 11178 (1995).

[11] A.H. MacDonald, T.M. Rice, W.F. Brinkman, Phys. Rev. 28, 3648 (1983).

[12] D.J. Thouless, J. Phys. C 18, 6211 (1985).

[13] C.W.J. Beenakker, H. van Houten, in: Solid State Physics - Advances in Research and Applications, Vol. 44, Eds. H. Ehrenreich, D. Turnbull, Academic Press, Boston 1991, p. 1.

[14] D.B. Chklovskii, B.I. Shklovskii, L.I. Glazman, Phys. Rev. B 46, 4026 (1992).

[15] C. Kallin, B.I. Halperin, Phys. Rev. B 30, 5655 (1984).

[16] S. Komiyama, Y. Kawaguchi, Phys. Rev. B 61, 2014 (2000).

[17] L. Eaves, S.T. Stoddart, R. Wirtz, A.C. Neumann, B.L. Gallagher, P.C. Main, M. Henini, Physica E 6, 136 (2000).

[18] B.E. Cole, J.M. Chamberlain, M. Henini, T. Cheng, W. Batty, A. Wittlin, J.A.A.J. Perenboom, A. Ardavan, A. Polisski, J. Singleton, Phys. Rev. B 55, 2503 (1997).

[19] F. Fang, W.E. Howard, Phys. Rev. Lett. 16, 797 (1966).

[20] A.H. MacDonald, J. Phys. C 18, 1003 (1985).

[21] A.M. Martin, K.A. Benedict, L. Eaves, submitted to Phys. Rev. Lett. 